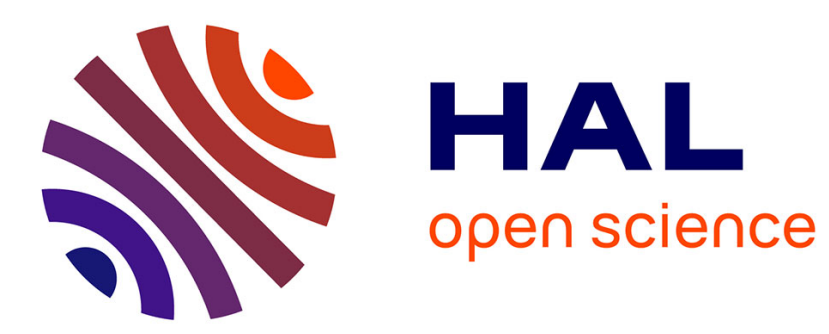

\title{
Dynamics in the presence of unilateral contacts and dry friction : a numerical approach
}

\author{
Michel Jean, Jean Jacques Moreau
}

\section{To cite this version:}

Michel Jean, Jean Jacques Moreau. Dynamics in the presence of unilateral contacts and dry friction: a numerical approach. Second Meeting on Unilateral Problems in Structural Analysis, Jun 1985, Prescudin, Italy. pp.151-196. hal-01864213

\section{HAL Id: hal-01864213 \\ https://hal.science/hal-01864213}

Submitted on 29 Aug 2018

HAL is a multi-disciplinary open access archive for the deposit and dissemination of scientific research documents, whether they are published or not. The documents may come from teaching and research institutions in France or abroad, or from public or private research centers.
L'archive ouverte pluridisciplinaire HAL, est destinée au dépôt et à la diffusion de documents scientifiques de niveau recherche, publiés ou non, émanant des établissements d'enseignement et de recherche français ou étrangers, des laboratoires publics ou privés. 


\title{
DYNAMICS IN THE PRESENCE OF UNILATERAL CONTACTS AND DRY FRICTION: A NUMERICAL APPROACH
}

\author{
M. Jean, J.J. Moreau \\ Laboratoire de Mécanique Générale des Milieux Continus \\ Université des Sciences et Techniques du Languedoc
}

\section{INTRODUCTION}

This paper is essentially devoted to mechanical systems with finite degree of freedom. However, as a test for adapting the proposed numerical methods to continuous systems, the representation of a one-dimensional medium by a finite chain of mass points is presented in fine.

The theoretical background has been defined by one of the authors in the Volume of the last meeting [1] and will only be recalled under some simplifying assumptions in the sequel. This background involves in particular that the possible shocks, occurring if two parts of the system suddenly enter into contact, are inelastic. The paper referred to was restricted to frictionless contacts ; in contrast dry friction is 
taken here into account, under the traditional law of Coulomb or some extension of it to anisotropic contacting surfaces. Mathematically, this law results in some non-smooth relation between the involved variables, i.e. not everywhere differentiable; the same is true for the expression of unilateral constraints. Treating both non-smooth aspects jointly is made possible here by using simple concepts of Convex Analysis, widely known today. Of course, some smooth dissipative actions - viscosity are easy to be introduced additionally.

Through the frictional effects as well as the inelasticity of possible shocks, irreversibility emerges as a dominant feature in the present approach.

No attention is paid in this paper to the existence and uniqueness study of the investigated motions. It has long been observed [2] [3] that when bodies exhibit dry friction in their possible points of contact, time intervals of smooth motion may end with instants at which some velocity jump occurs necessarily; this is called a tangential shock. Sufficient conditions imposed to the system constants and to initial data have recently been given [4], in order that the motion begins with a time interval exempt from such an accident (and from contact breaking off as wel1); thereby a local existence and uniqueness theorem has been obtained. Since the algorithms presented in the sequel are devised to treat shocks on an equal footing with regular motions, they prove able to include such events in the computation.

An example presented in Sect. 8 is that of the chattering motion of a solid body elastically driven along a plane wall (a piece of chalk pushed at an angle against a blackboard). Oscillations with intermittent 
breaking of contact are exhibited. The slip velocity may drop recurrently to zero ; contrary to common belief, this "stick-slip" phenomenon is not essentially connected with the possible dependence of the friction coefficient on slip velocity. Anyway, the proposed algorithm allows one to take such a dependence into account, even under its extreme form : distinguishing between the "static" (i.e. for zero slip velocity) and "dynamic" friction coefficients.

Another example presented in section 6 is that of the motion of a chain of material points in contact with a wall moving at constant velocity along one of the axes. The points are connected together by springs and dashpots and each of them is pushed against the wall by a single pair of springs and dashpots. The points are submitted to Coulomb friction from the wall. The example presented shows a "stick-slip" phenomenon. 


\section{ANALYTICAL SETTING}

As usual in Analytical Dynamics, the totality of the possible positions of the system $S$, regardless of the unilateral constraints which will be imposed further, constitutes a differential manifold $Q$, with dimension $l$ equal to the freedom of $S$. Let $\left(q^{i}\right), i=1,2, \ldots, l$ denote (at least local) coordinates in 2 . For every differentiable motion $t \rightarrow q(t)$, the derivatives $\dot{q}^{i}(t)$ are the components of the velocity $\dot{q}(t)$, an element of the tangent space to $Q$ at the point $q(t)$. The kinetic energy of $S$ is supposed expressed as a timeindependent positive definite quadratic form of $\dot{q}$, namely

$$
T(q, \dot{q})=\frac{1}{2} a_{i j}(q) \dot{q}^{i} \dot{q}^{j}
$$

where $a_{i j}=a_{j i}$ are known functions of $q=\left(q^{l}, \ldots, q^{l}\right)$.

For simplicity we shall make in the whole paper the following assumptions.

ASSUMPTION $1 . S$ is partitioned into a finite collection of subsystems $\left(S_{\alpha}\right), \alpha=1, \ldots, \pi$, which may interact through configuration-dependent and/or velocity-dependent forces, but without any mutual hinge or contact. Therefore $Q$ equals the product of the corresponding manifolds $q$ and $\mathrm{T}(\mathrm{q}, \dot{\mathrm{q}})$ splits into the sum of the respective kinetic energies, namely 
for subsystem $S_{\alpha}$

$$
\mathrm{T}_{\alpha}\left(\mathrm{q}_{\alpha}, \dot{\mathrm{q}}_{\alpha}\right)=\frac{1}{2} \mathrm{a}_{\mathrm{mn}}^{\alpha}\left(\mathrm{q}_{\alpha}\right) \dot{\mathrm{q}}_{\alpha}^{\mathrm{m}} \dot{\mathrm{q}}_{\alpha}^{\mathrm{n}}
$$

Here $\left(q_{\alpha}^{1}, q_{\alpha}^{2}, \ldots\right)$ denote (at least local) coordinates of the point $q_{\alpha}$ in the manifold $?_{a}$. Unlike Latin indices, the repetition of Greek indices will not be understood as implying summation.

ASSUMPTION 2 . For every $\alpha$, it is supposed that, at least locaily in manifold $\mathcal{Q}_{\alpha}$, the coordinate system $\left(q_{\alpha}^{1}, q_{\alpha}^{2}, \ldots\right)$ may be constructed in such a way that the coefficients $a_{m n}^{\alpha}$ in (2.2) do not depend on $q_{\alpha}$. This means that the Riemann metric defined on manifold $q_{\alpha}$ by the following expression of arc-length ds

$$
\mathrm{ds}^{2}=\mathrm{a}_{\mathrm{mn}}^{\alpha}\left(\mathrm{q}_{\alpha}\right) \mathrm{dq}_{\alpha}^{\mathrm{m}} \mathrm{dq_{ \alpha } ^ { \mathrm { n } }}
$$

actually turns out to be Euclidean.

Such is trivially the case if subsystem $S_{\alpha}$ consists of a single mass-point moving through some region of the physical space.

In another usual case, $S_{\alpha}$ will consist in a rigid body, allowed to perform only motions parallel to some fixed plane. Locating each of its possible positions by the Cartesian (orthonormal) coordinates X,Y of the center of mass $G$ and by the rotation angle $\theta$, one obtains

$$
\mathrm{T}_{\alpha}=\frac{1}{2} \mathrm{~m}\left(\dot{\mathrm{X}}^{2}+\dot{\mathrm{Y}}^{2}\right)+\frac{1}{2} \mathrm{I} \dot{\theta}^{2} .
$$

Here $m$ denotes the mass of the rigid body and $I$ its moment of inertia about the axis drawn through G, normal to the plane of motion. This makes $\left[a_{m n}^{\alpha}\right]$ a diagonal matrix, constant with regard to the triplet 


$$
\left(q_{\alpha}^{1}, q_{\alpha}^{2}, q_{\alpha}^{3}\right)=(X, Y, \theta)
$$

As already said, the investigated unilateral constraints are not taken into account at the stage of defining the parametrization of $S$. Such superimposed constraints will consist in impenetrability effects about which we shall make now some simplifying hypotheses.

ASSUMPTION 3 . Unilateral contacts never occur between two different members of the previously defined partition; they take place either inside some $S_{\alpha}$ or between some of these subsystems and some external body with prescribed motion. In addition, it is supposed that the geometric effect of impenetrability upon the concerned subsystem $S_{\alpha}$ is expressed by a single scalar inequality

$$
f_{\alpha}\left(t, q_{\alpha}^{1}, q_{\alpha}^{2}, \ldots\right) \leqslant 0
$$

where the real function $\mathrm{f}_{\alpha}$ is $\mathrm{C}^{1}$, with nonzero gradient (at least in a neighborhood of the hypersurface $f_{\alpha}=0$ ). This inequality defines for every $t$ a closed subset $L_{\alpha}$ of manifold $\gamma_{\alpha}$.

Under these assumptions, let us first write the Lagrange equations governing the motion of the system $S$ during a time interval of smooth motion, i.e. an interval, if any, where the functions $t \rightarrow q(t)$ happen to be twice differentiable. Since the matrix $a_{m n}^{\alpha}$ has been supposed constant with regard to $t$ and to $q_{\alpha} \simeq\left(q_{\alpha}^{1}, q_{\alpha}^{2}, \ldots\right)$, the Lagrange equations relative to subsystem $S_{\alpha}$ reduce to

$$
a^{\alpha} \ddot{q}_{\alpha}=r^{\alpha}+Q^{\alpha}(t, q, \dot{q})
$$

The writing on the left-hand side is that of product matrix, i.e. the 
covector with components $a_{\operatorname{mn}_{\alpha}^{\alpha}}^{\alpha} \ddot{q}^{n}$. The covector $r^{\alpha} \stackrel{\simeq}{-}\left(r_{1}^{\alpha}, r_{2}^{\alpha}, \ldots\right)$ represents the force of constraint arising from the possible unilateral contact experienced by $S_{\alpha}$; the function $Q^{\alpha} \simeq\left(Q_{1}^{\alpha}, Q_{2}^{\alpha}, \ldots\right)$ expresses all other forces, a priori depending on the position and velocity of the whole system $S$ and possibly also on $t$ as independent variable, that subsystem $S_{\alpha}$ undergoes from any part. It is only through such terms as $Q^{\alpha}$ that the Lagrange equations corresponding to the respective members $S_{\alpha}$ of the partition of $S$ are coupled together.

Under the writing (2.5) is summarized a set of scalar equations whose number equals the freedom of subsystem $S_{\alpha}$. There is to join with them the geometric inequality (2.4) and some mechanical information we shall state in the section to come, about the force of unilateral constraint $r^{\alpha}$. 


\section{LAWS OF FRICTIONAL UNILATERAL CONTACT}

The primary statement concerning the force of constraint $r^{\alpha} \simeq\left(r_{1}^{\alpha}, r_{2}^{\alpha}, \ldots\right)$, introduced in $(2.5)$, is that it vanishes at every instant $t$ where the corresponding contact is not in effect, i.e. when the position $q_{\alpha}(t) \stackrel{\simeq}{=}\left(q_{\alpha}^{1}(t), q_{\alpha}^{2}(t), \ldots\right)$ verifies $(2.4)$ as a strict inequality.

On the contrary, every position $q_{\alpha}$ of $S_{\alpha}$ such that (2.4) holds as an equality corresponds to effective contact. The governing laws have to be made precise in the framework of the 3-dimensional physical space; afterwards in sect. 4 we shall transcribe them in terms of analytical variables.

Let us first consider the case where the investigated contact takes place between some part $B_{1}$ of $S_{\alpha}$ and an external body $B_{0}$ with prescribed motion. Such a contact will always be supposed located at a single point $M$ and the definition of a plane is assumed, which will be declared a common tangent plane at the point $M$ to both boundaries of $B_{0}$ and $B_{1}$. This does not preclude bodies presenting edges or vertices; one of them may even reduce to a single material point, provided the boundary of the other one is a regular surface. Anyway, let us denote by $M_{0}$ and $M_{1}$ the respective particles of $B_{0}$ and which happen to lie at point $M$ at 
the instant in view.

The contact actions exerted by $B_{0}$ upon $B_{1}$ are supposed represented by a single force $R$ applied to particle $M_{1}$; hence any torque expressing "resistance to rolling" is neglected. Kinematically, for every imagined differentiable motion of the system, passing at time $t$ by the considered position, the difference of the velocity vectors of $M_{0}$ and $M_{1}$,

$$
u=V\left(M_{1}\right)-V\left(M_{0}\right)
$$

is referred to as the slip velocity of $B_{1}$ relative to $B_{0}$.

Let us denote by $E_{3}$ the linear space of the vectors of physical space. Let $N \in E_{3}$ be the unit vector normal to the common tangent plane at point $M$, directed toward $B_{1}$.

This Section is devoted to motions smooth enough for Lagrange's equations to make sense. The existence of accelerations is then postulated; therefore velocities are continuous functions of time. Elementary kinematics entails in that case that the slip velocity satisfies

$$
N \cdot U=\dot{U},
$$

i.e. $U$ belongs to the two-dimensional subspace $T$ of $E_{3}$, tangent to both contacting bodies.

The admitted law of dry friction stipulates a relation between the elements $U \in T$ and $R \in E_{3}$. Here are two equivalent formulations, generalizing to possibly anisotropic contacting surfaces the traditional law of Coulomb. 
DECOMPOSED FORM (see more details in [5] [6]).

The reaction vector $R$ lets itself be uniquely decomposed into

$$
R=R_{T}+R_{N} N \quad, \quad R_{T} \in T, \quad R_{N} \in \mathbb{R} .
$$

It is first stated that the real number $R_{N}$ satisfies

$$
R_{N} \geqslant 0 \quad,
$$

i.e. no adhesive effect takes place between the bodies in contact.

Secondly there is given a closed convex subset $D_{1}$ of $T$, containing the zero of $E_{3}$. For every $R_{N} \geqslant 0$ put

$$
D\left(R_{N}\right)=R_{N} D_{1}
$$

and denote by $\psi$ the indicator function of the subset $D\left(R_{N}\right)$ of $T$, i.e.

$$
\psi(X)= \begin{cases}0 & \text { if } \quad x \in D\left(R_{N}\right) \\ +\infty & \text { if } x \in T \backslash D\left(R_{N}\right) .\end{cases}
$$

This is a lower semi-continuous convex function defined in $T$. Recall that for every $x \in T$ the subdifferential $\partial \psi(X)$, relative to the Euclidean scalar product in $T$, equals the set of the elements $y$ of $T$ which are outward normal to the closed convex set $D\left(R_{N}\right)$ at point $x$ in the conventional sense of Convex Analysis. This subdifferential is empty if $x \notin D\left(R_{N}\right)$; otherwise it contains at least the zero of $T$ and reduces to this single element in case $X$ is interior to $D\left(R_{N}\right)$.

Then the expected relation writes down as 
$-u \in \partial \psi\left(R_{T}\right)$.

For every fixed $R_{N}$ this is a resistance law of the rate-independent sort, also familiar in Plasticity theory ; some equivalent forms are classically given to such laws, in terms of a "principle of maximal dissipation" or also in terms of a "dissipation function" (actually the support function of the set $\left.-D\left(R_{N}\right)\right)$; see e.g. [6] [10].

The traditional law of Coulomb for isotropic contact is simply obtained by taking as $D_{1}$ the closed disk in $T$, with center at the origin and radius equal to the friction coefficient.

CONICAL FORM.

Standard arguments of Convex Analysis [7] permit to translate the above formulation into a statement avoiding the decomposition (3.3) .

Let us denote by $C$ the closed convex cone in $E_{3}$ (with vertex at the origin) generated by the set $D_{1}+N$. In the special case of isotropic Coulomb friction, this set is the closed region delimited in $E_{3}$ by what is traditionally called the cone of friction : a conical surface of revolution about vector $N$.

Then the previous formulation is found equivalent to

$-u \in \operatorname{proj}_{T} \partial \psi_{C}(R)$.

Here $\operatorname{proj}_{T}$ denotes the orthogonal projection mapping from $E_{3}$ onto $T$. In the sense of Convex Analysis in $E_{3}$, the set $\partial \psi_{C}(R)$ constitutes the outward normal cone to $C$ at the point $R$. Since this set is empty when $R \notin C$, one observes in particular that the no-adhesion assumption (3. 4) is involved in such a writing. 
What precedes has been presented for a part $B_{1}$ of $S_{\alpha}$ in contact with some external body. The formulation is quite similar in the case where equality $\mathrm{f}_{\alpha}=0$ expresses the contact, occuring at some point $M$ of physical space, between two bodies $B_{1}$ and $B_{2}$, both parts of subsystem $S_{\alpha}$. Again the definition of a common tangent plane at $M$ to the respective boundaries is assumed; $M_{1}$ and $M_{2}$ denote the respective particles of $B_{1}$ and $B_{2}$ which happen to lie at $M$, at the considered instant, and $U$ the slip velocity of $B_{1}$ relative to $B_{2}$, i.e.

$$
u=V\left(M_{1}\right)-V\left(M_{2}\right)
$$

The contact actions exerted by $B_{2}$ upon $B_{1}$ are supposed represented by a single force $R$ applied to $M_{1}$; hence, in view of Newton's third law, the contact actions exerted by $B_{1}$ upon $B_{2}$ are represented by the single force $-R$ applied to $M_{2}$.

The law of dry friction stipulates a relation between $R$ and $U$, exactly in the same terms as in the preceding case.

Let us finally take notice of the simplifying circumstance of twodimensional contact. In fact, it may happen that, due to the primitive constraints of the system (i.e. the perfect constraints which have permitted its parametrization) the set of the values of $V\left(M_{1}\right)-V\left(M_{2}\right)$ (resp. $V\left(M_{1}\right)$ in case of external contact) which, regardless to contact permanence, are possible, is not the whole of $E_{3}$ but only a twodimensional linear subspace $E_{2}$, a priori depending on $q_{\alpha}$. Then the role of $T$ is played by some one-dimensional subspace of $E_{2}$; the cone $C$ simply reduces to an angular region whose boundary consists of 
two half-lines $D_{1}$ and $D_{2}$ (possibly non-symmetric with respect to the normal direction $N$ : this reflects difference in the friction coefficients associated with either directions of sliding).

The active part of the reaction $R$ consists now in the orthogonal projection of this vector on $E_{2}$. Similar formulations as above then hold, with $E_{3}$ replaced by $E_{2}$, but two-dimensionality brings the following simplification.

Let $A_{1}$ and $A_{2}$ denote generators of $D_{1}$ and $D_{2}$. The vector $B=A_{2}-A_{1}$ cannot be parallel to $N$; then, for every $u \in T$, is readily found equivalent to
$R \in C$
$R \in D_{1} \quad$ if $\quad$ B. $U>0$
$R \in D_{2} \quad$ if $\quad B . U<0$ 


\section{EQUATIONS OF SMOOTH MOTIONS}

The law of frictional unilateral contact formulated above has now to be transcribed into the analytical setting of Sect. 2 .

Let us first consider the case of the contact occurring between a part $B_{1}$ of $S_{\alpha}$ and some external body $B_{0}$. The contacting particle $M_{1}$ of $B_{1}$ a priori depends on the position $q_{\alpha} \in Q_{\alpha}$ attained by the system $S_{\alpha}$ at the considered instant and also, since $B_{0}$ has been assumed to possess a certain prescribed motion, on the time $t$ as independent variable. Supposing $t$ fixed and given the position $q_{\alpha}(t) \in \mathcal{Q}_{\alpha}$, let us consider a chain of positions of $S_{\alpha}$ depending in a differentiable way on some real variable $\tau$. Using local coordinates $\left(q_{\alpha}^{1}, q_{\alpha}^{2}, \ldots\right)$ in $Q_{\alpha}$, define it by

$$
q_{\alpha}^{i}=\kappa^{i}(\tau)
$$

with real functions $\tau \mapsto \kappa^{i}(\tau), i=1,2, \ldots$ such that $\kappa^{i}(0)=q_{\alpha}^{i}(t)$; this is conventionally called a virtual displacement at fixed time $t$. If some particle $P$ of $S_{\alpha}$ has been identified, every value of $q_{\alpha}$ yields a position of $P$, relatively to the reference frame $F$ adopted in physical space. When $\mathrm{q}_{\alpha}$ is expressed by (4.1) as a function of $\tau$, the said position of $P$ becomes a $\tau$-dependent point in $F$ that we shall 
denote by $\tau \rightarrow P^{F}(\tau)$. Analysing the geometry of the system and the way parameters $\left(q_{\alpha}^{1}, q_{\alpha}^{2}, \ldots\right)$ have been defined, one may obtain for the vector derivative $\frac{\delta P F}{\delta \tau}(0)$ an expression in which the real derivatives $\frac{\delta \kappa}{\delta \tau}(0)$ figures linearly (using here $\delta$ instead of $d$ to denote differentials is traditional in order to prevent confusion between virtual displacements and proper motions). This is true in particular if $P$ equals the particle $M_{1}$ identified above ; one obtains an expression of the form $\frac{\delta M_{1}^{F}}{\delta \tau}=G\left(\frac{\delta K}{\delta \tau}\right)$.

Here $\frac{\delta \kappa}{\delta \tau}$, with components $\frac{\delta \kappa^{i}}{\delta \tau}(0)$, constitutes an element of the tangent space $E_{\alpha}$ to the manifold $\bigcap_{\alpha}$ at point $q_{\alpha}(t)$ and $G$ denotes a certain linear mapping of $E_{\alpha}$ into $E_{3}$.

This calculation is first used to express the covariant components (or "generalized components") of the force $R$ exerted on the particle $M_{1}$ of $B$. By definition these components are the real numbers $r_{1}, r_{2}, \ldots$ such that, for every virtual displacement as above,

$$
R \cdot \frac{\delta M_{1}^{F}}{\delta \tau}=r_{i} \frac{\delta \kappa^{i}}{\delta \tau} \text {. }
$$

The real numbers $r_{i}$ may be viewed as the components of some element $r$ of the dual of $E_{\alpha}$, the linear space $E_{\alpha}^{*}$ cotangent to the manifold $\eta_{\alpha}$ at point $\mathrm{q}_{\alpha}(\mathrm{t})$. Denoting by $<.$, . > the bilinear pairing between $\mathrm{E}_{\alpha}$ and $E_{\alpha}^{\star}$ and using (4.2), we write equivalently

$$
R \cdot G\left(\frac{\delta K}{\delta \tau}\right)=\left\langle r, \frac{\delta K}{\delta \tau}\right\rangle \text {. }
$$

Since the choice of functions $\tau \rightarrow \kappa^{i}(\tau)$ allows one to identify 
with an arbitrary element of $\mathrm{E}_{\alpha}$, this is equivalent to

$$
r=G^{*}(R) \text {, }
$$

where $G^{\star}: E_{3} \rightarrow E_{\alpha}^{\star}$ is the transpose of the linear mapping $G: E_{\alpha} \rightarrow E_{3}$ (the space $E_{3}$ of the vectors of the physical space is paired with itself through the Euclidean scalar product).

The same linear mapping $G$ is involved in the calculation of the velocity of particle $M_{1}$ in the course of some motion of $S_{\alpha}$. Such a motion is defined by giving the position $q_{\alpha} \in \mathcal{Z}_{\alpha}$, i.e. the local coordinates $q_{\alpha}^{1}, q_{\alpha}^{2}, \ldots$, as differentiable functions of time. Then, for every identified particle $P$ of the system, the position of $P$, relative to the reference frame $F$ becomes a $t$-dependent point of this frame, whose vector derivativeconstitutes the velocity vector $V(P)$. For simplicity, we restrict ourselves in the sequel to scleronomic systems, i.e. the position of $P$ in $F$ is determined by $q_{\alpha} \underline{\sim}\left(q_{\alpha}^{1}, q_{\alpha}^{2}, \ldots\right)$ independently of time; this is in fact the only practical case where the expression of kinetic energy reduces to the form assumed in (2.1). Then expressing $V(P)$ from the t-derivatives $\dot{q}_{\alpha}^{1}, \dot{q}_{\alpha}^{2}, \ldots$ requires exactly the same calculation as was made above for expressing $\delta \mathrm{P} / \delta \tau$ in some virtual displacement, from the $\tau$-derivatives $\frac{\delta K^{l}}{\delta \tau}, \frac{\delta \kappa^{2}}{\delta \tau}, \ldots$ (in the absence of the scleronomy assumption, there would appear in $V(P)$ some additional term). Hence, by making $P$ equal to the particle $M_{1}$ one obtains

$$
V\left(M_{1}\right)=G\left(\dot{q}_{\alpha}\right)
$$

The contacting particle $M_{0}$ of external body $B_{0}$ a priori depends on the position $q_{\alpha} \in Q_{\alpha}$ of $S_{\alpha}$ and also on $t$ as independent variable, 
since, by assumption, $B_{0}$ exhibits some given motion. Hence the velocity $V\left(M_{0}\right)$ appears as a vector function $u_{e}$ of $\left(t, q_{\alpha}^{1}, q_{\alpha}^{2}, \ldots\right)$ which has to be calculated from geometric inspection. This finally yields an expression of the slip velocity (3.1) under the form

$$
u=G\left(\dot{q}_{\alpha}\right)+u_{e}\left(t, q_{\alpha}\right)
$$

Of course the linear mapping $G: E_{\alpha} \rightarrow E_{3}$ depends on $q_{\alpha} \in \overbrace{\alpha}$ and also on time $t$ as independent variable, since these elements are needed in order to identify the contact particle $M_{1}$.

What precedes makes sense only for $t$ and $q_{\alpha}$ such that the contact is effective, i.e. inequality $(2.4)$ holds as an equality. In the sequel we shall need the definition of $G$ and $U_{e}$ to be extended in a smooth arbitrary way to every $\left(t, q_{\alpha}\right)$, at least in some neighborhood of hypersurface $f_{\alpha}\left(t, q_{\alpha}\right)=0$.

The case of contact occurring between two parts $B_{1}$ and $B_{2}$ of $S_{\alpha}$ lets itself be treated in the same way. Then both opposite reactions $R$ and $-R$ experienced by the contact particles $M_{1}$ and $M_{2}$ are forces acting on the system. Thus the analytical treatment will involve the covariant components of this pair of forces, i.e. the real numbers $r_{1}, r_{2}, \ldots$ such that, instead of (4.3) one has

$$
R \cdot \frac{\delta M_{1}^{F}}{\delta \tau}-R \cdot \frac{\delta M_{2}^{F}}{\delta \tau}=r_{i} \frac{\delta K^{i}}{\delta \tau}
$$

for every virtual displacement. Here a linear mapping $G: \mathrm{E}_{\alpha} \rightarrow E_{3}$ may be found, such that

$$
\frac{\delta M_{1}^{F}}{\delta \tau}-\frac{\delta M_{2}^{F}}{\delta \tau}=G\left(\frac{\delta K}{\delta \tau}\right) .
$$


This finally yields the same writing as in (4.4) . Besides, supposing again the system scleronomic, one finds as expression of the slip velocity $U=V\left(M_{1}\right)-V\left(M_{2}\right)$

$$
u=G\left(\dot{q}_{\alpha}\right),
$$

which is the same as (4.5), with $u_{e}=0$.

It is now easy to list a complete set of equations governing any possible smooth motion of the system. This consists in writing, for every subsystem $S_{\alpha}$, the Lagrange equations (2.7), the expression (4.5) or (4.6) of the slip velocity $U$, the expression (4.4) of $r$ from the reaction $R$ and, finally, the law of frictional contact as stated in Sect. 3 . Let us symbolize the latter as

$$
R \in \operatorname{cou}_{\alpha}\left(t, q_{\alpha}, u\right) \quad .
$$

Here the right-hand member represents the subset of $E_{3}$ (resp. $E_{2}$ in the case of two-dimensional contact) consisting of the values of the contact reaction $R$ which agree with the formulated dry friction law if, the position of $S_{\alpha}$ being $q_{\alpha}$ at time $t$, the slip velocity happens to assume the value $U$. It is an essential feature of this law that it cannot be "solved" in order to express $R$ as a single-valued function of $U$ (nor $U$ as a single-valued function of $R$ ). Instead, the symbol cou $_{\alpha}$ represents a multivalued mapping or multifunction that we propose to call the Coulomb multifunction associated with the possible contact affecting subsystem $S_{\alpha}$.

The description of friction, in sect. 3 , was developed under such smoothness assumptions that $U$ essentially belongs to the linear subspace 
$T$ of $E_{3}$, tangent to contacting bodies. Let us put explicitely :

CONVENTION 1 . In the case of contact, i.e. $f_{\alpha}\left(t, q_{\alpha}\right)=0$, the value of the multifunction $\operatorname{cou}_{\alpha}$ is defined equal to the empty set whenever $u \notin T$.

In other words, the existence of some $R$ verifying (4.7) implies $u \in T$

Furthermore, the no-contact case $f\left(t, q_{\alpha}\right)>0$ may be embodied in the same writing by putting :

CONVENTION 2. In the case $f_{\alpha}\left(t, q_{\alpha}\right)>0$, the value of the multifunction $\operatorname{cou}_{\alpha}$ is declared to be the singleton $\{0\}$ whichever is $U$ in $E_{3}$.

Incidentally, the geometric impenetrability condition could also be included by agreeing that the multifunction assumes the value $\emptyset$ whenever it is not satisfied. We shall avoid this trick for it does not prove convenient when discussing numerical approximation.

In exploiting the above set of conditions, one may wish to eliminate the variables $R$ and $U$. This finally yields

$$
a^{\alpha} \ddot{q}_{\alpha}-Q^{\alpha}(t, q, \dot{q}) \in G^{*}\left(\operatorname{cou}_{\alpha}\left(t, q_{\alpha}, G\left(\dot{q}_{\alpha}\right)+u_{e}\left(t, q_{\alpha}\right)\right)\right)
$$

a second order differential inclusion.

On the contraryone may keep the reactions as primary unknowns. One has the following set of equations :

- if $\alpha$ is the index of a subsystem with contact at time $t$ 


$$
\begin{aligned}
& a^{\alpha} \ddot{q}_{\alpha}=Q_{\alpha}(t, q, \dot{q})+G_{\alpha}^{\star}\left(R_{\alpha}\right) \\
& u_{\alpha}=G_{\alpha}\left(\dot{q}_{\alpha}\right)+u_{e \alpha} \\
& R_{\alpha}=R_{T}+R_{N_{\alpha}} N_{\alpha} \\
& R_{N_{\alpha}} \geqslant 0 \\
& -u_{\alpha} \in \partial \psi\left(R_{T}\right)
\end{aligned}
$$

where $\partial \psi$ is the subdifferential of the indicator function of the convex set $D\left(R_{N_{\alpha}}\right)=R_{N_{\alpha}} D_{1}$;

- if $\alpha$ is the index of a subsystem with no contact

$$
a^{\alpha} \ddot{q}_{\alpha}=Q_{\alpha}(t, q, \dot{q})
$$

The initial conditions $q\left(t_{0}\right), \dot{q}\left(t_{0}\right)$ being fixed, let us write as

$$
u_{\alpha}=A_{\alpha}(R) \quad, \quad R=\left(R_{1}, \ldots, R_{\alpha}, \ldots\right)
$$

the result of the elimination of $q_{\alpha}$ between (4.9) and (4.10). This defines the mapping $A_{\alpha}$, under the usual assumptions ensuring existence and uniqueness for the solution to the differential equations (4.9).

Furthermore, the inclusion (4.13) may equivalently be written as a variational inequality, expressing the "principle of maximal dissipation", namely

$$
R_{T_{\alpha}} \in D\left(R_{N_{\alpha}}\right) \text { and } \forall \Phi \in D\left(R_{N_{\alpha}}\right),\left(\Phi-R_{T_{\alpha}}\right) \cdot u_{\alpha} \geqslant 0
$$

where the dot refers to scalar product in $E_{3}$.

$$
\text { Hence conditions (4.9) to (4.13) are finally equivalent to a }
$$


system of quasi-variational inequalities.

In usual cases, due to the positiveness of inertia matrices and the convexity of elastic potential, the mapping $A \simeq\left(A_{1}, \ldots, A_{\alpha}, \ldots\right)$ turns out to be monotone in the sense of Minty and the mapping Id - $\rho A$ is a contraction for every $\rho>0$ in some interval.

Finally the problem may be formulated as the following fixed point condition

$$
R_{T_{\alpha}}=\operatorname{proj}_{D\left(R_{N_{\alpha}}\right)}\left(R_{T_{\alpha}}-\rho A_{\alpha}(R)\right)
$$

together with $(4.11),(4.12)$. 
5. ALGORITHM OF CONICAL TYPE

By introducing the additional unknown vector function of $t$

$$
u_{\alpha}=\dot{q}_{\alpha},
$$

one gives to the differential inclusion (4.8) the form

$$
a^{\alpha} \dot{u}_{\alpha}-Q^{\alpha}(t, q, \dot{q}) \in G^{*}\left(\operatorname{cou}_{\alpha}\left(t, q_{\alpha}, G\left(u_{\alpha}\right)+u_{e}\left(t, q_{\alpha}\right)\right)\right) \text {. }
$$

Let $t_{0}, t_{1}, \ldots, t_{i}, \ldots$ be a time discretization, with mesh length $t_{i+l}-t_{i}=h$ independent of $i$ for simplicity. Dropping the subscript $\alpha$ which refers to the considered member of the partition of $S$, we shall denote by $q_{i}, u_{i}$ some approximants of vectors $q_{\alpha}\left(t_{i}\right), u_{\alpha}\left(t_{i}\right)$. Then (5.1) is discretized into

$$
q_{i+1}=q_{i}+h u_{i}
$$

The discretization of (5.2) has to be performed in such a way that each step, starting with known $\mathrm{q}_{i}$ and $u_{i}$, yields a computable value of $u_{i+1}$. In simple cases, this value is expected to be uniquely defined (anyway, it has long been recognized [2] that, in the dynamics of systems with dry friction, uniqueness of the motion consequent to initial data is not granted in general). 
Recall that $q, \dot{q}$ in $Q^{\alpha}$, on the left-hand side of (5.2), refer to the whole system $S$ and not only to the member $S_{\alpha}$ of the partition. When computing an approximant of $Q^{\alpha}$, one may assign to $t$ and $q$ in this function the starting values of the considered step or, alternatively, some anticipated values such as $q_{i+l}$ or $\left(q_{i}+q_{i+1}\right) / 2$; the influence of this choice upon numerical precision is discussed in [7]. As for $\dot{q}$ in $Q^{\alpha}$, we decide to adopt the starting values of the step ; in that respect, our computation looks like an "implicit" scheme : this is justified by the assumed smoothness of the function $Q^{\alpha}$. For the sequel, let us denote by $Q_{i}^{\prime}$ the approximant of vector $Q^{\alpha}$ constructed in that way.

Incidentally, in the absence of assumption 2 , made in sect. 2 for simplicity, there would appear in the left-hand members of Lagrange equations some quadratic terms in $\dot{q}$ : the same would apply to them. Similarly, the linear mapping $G$ of $E_{\alpha}$ to $E_{3}$ and the velocity $u_{e} \in E_{3}$ of a possible external obstacle, which are smooth known functions of $t$ and $q$, will be calculated from the starting values $t_{i}$, $q_{i}$ or through some anticipation as above.

In contrast, the Coulomb multifunction behaves critically since, with regard to variables $t$ and $q_{\alpha}$, it is discontinuous on the boundary of the permitted region. Strictly speaking, $\operatorname{cou}_{\alpha}$ is not defined if $f_{\alpha}\left(t, q_{\alpha}\right)>0$; however, in the course of computation, a certain amount of violation of the unilateral constraint may be faced, so we agree to extend in a smooth arbitrary way the definition of $\operatorname{cou}_{\alpha}$, at least when condition $f_{\alpha}\left(t, q_{\alpha}\right) \leqslant 0$ is moderately violated. For instance, in 
the case of the traditional (isotropic) Coulomb law, a cone of revolution in $E_{3}$ will be considered, with axis and angle depending continuously on $t$ and $q_{\alpha}$, so as to reduce to the proper Coulomb cone when the contact condition $f\left(t, q_{\alpha}\right)=0$ holds. The definition of $\operatorname{cou}_{\alpha}$ derives from this cone in the way explained in sect. 3 .

Recall that $\operatorname{cou}_{\alpha}\left(t, q_{\alpha}, U\right)=\{0\}$ if $f_{\alpha}\left(t, q_{\alpha}\right)<0$. Hence every discretization step rests on the consideration of some test point $\left(t_{i}^{\prime}, q_{i}^{\prime}\right)$; an expedient choice proves to be $t_{i}^{\prime}=t_{i}+h / 2$, $q_{i}^{\prime}=q_{i}+h u_{i} / 2$.

If $f_{\alpha}\left(t_{i}^{\prime}, q_{i}^{\prime}\right)<0$ it will be admitted that $\operatorname{cou}_{\alpha}=\{0\}$ for the considered step, so that (5.2) simply discretizes into

$$
a\left(u_{i+1}-u_{i}\right) / h-Q_{i}^{\prime}=0
$$

corresponding to the dynamics of the system in the absence of contact. Since $a$ is an invertible matrix, this yields as end of the computation step

$$
u_{i+1}=u_{i}+h a^{-1} Q_{i}^{\prime} .
$$

In any case, we shall denote by $u_{i}^{\prime}$ the left member of (5.5). Furthermore let $g$ be the gradient of $f_{\alpha}$ at the test point, i.e. the element of $E_{\alpha}^{*}$ with components $\partial f_{\alpha} / \partial q_{j}^{\alpha}$.

For simplicity, suppose the junction $f_{\alpha}$ constant in $t$ and $u_{e}=0$. When $f_{\alpha}\left(q_{i}^{\prime}\right) \geqslant 0$, computation goes on as follows. If $\left\langle u_{i}^{\prime}, g\right\rangle \leqslant 0$ we estimate that motion takes place without contact action and adopt again (5.5) as step end. 
If, on the contrary, $\left\langle u_{i}^{\prime}, g\right\rangle>0$, the nontrivial multifunction $u \rightarrow \operatorname{cou}_{\alpha}\left(t, q_{\alpha}, u\right)$ has to be considered, with $\left(t, q_{\alpha}\right)$ conveniently fixed at the same test point $\left(t_{i}^{\prime}, q_{i}^{\prime}\right)$ as above, and shortly written cou $(U)$. In order to yield a precise characterization of $u_{i+1}$, the discretization has to involve this multifunction through some "implicit" procedure, i.e. the value substituted for $u_{\alpha}$ in the right-hand side of (5.2) will be the unknown $u_{i+1}$ itself. Since the set cou(U) is essentially conic, one may equivalently multiply both members by the positive number h, thus obtaining

$$
a\left(u_{i+1}-u_{i}^{\prime}\right) \in G^{*}\left(\operatorname{cou}\left(G\left(u_{i+1}\right)\right)\right) \text {. }
$$

We place ourselves in the regular case where the linear maipping $G$ : $E_{\alpha} \rightarrow E_{3}$ (resp. $E_{2}$ in bidimensional cases) is surjective, i.e. every value $U=G\left(\dot{q}_{\alpha}\right)$ in $E_{3}$ (resp. $E_{2}$ ) of the relative velocity of contacting bodies at point $M$, without regard to contact permanence nor impenetrability, may be obtained by choosing suitably the time-derivative $\dot{\mathrm{q}}_{\alpha} \in \mathrm{E}_{\alpha}$. Then the transpose $\mathrm{G}^{*}$ is injective ; in addition, the following geometrical remark will be of use.

If contact holds in the considered position of the system, a value $u$ of $\dot{q}_{\alpha}$ is compatible with further preservation of condition $f_{\alpha}\left(q_{\alpha}(t)\right) \leqslant 0$ if $\langle u, g\rangle \leqslant 0$. Now $f_{\alpha} \leqslant 0$ expresses that, in physical space, the subsequent imagined motion does not make the bodies interpenetrate. This proves the implication

$$
\left.\langle u, g\rangle \leqslant 0 \Rightarrow G(u) \cdot N \geqslant 0 \quad \text { (i.e. }\left\langle u, G^{*}(N)\right\rangle \geqslant 0\right)
$$

which, in view of the unilateral version of Lagrange multiplier theorem, 
is equivalent to

$$
\exists \lambda \geqslant 0: G^{\star}(N)=-\lambda g \text {. }
$$

Actually $\lambda>0$, due to injectivity of $G^{\star}$. This shows in particular that the element $U=G(u)$ lies in the common tangent space $T$ to contacting bodies if and only $u$ belongs to $T$, the subspace of $E_{\alpha}$ orthogonal to the element $g$ of $\mathrm{E}_{\alpha}^{\star}$.

How inclusion (5.6) may determine $u_{i+1}$ will only be demonstrated here by the simple example of two-dimensional friction, as formulated at the end of sect. 3 (another very clear instance, developed in [7], is that of a single mass-point, unilaterally confined by some moving boundary).

The cone $C$ in $E_{2}$ is generated by a couple of elements $A_{1}, A_{2}$. Thus its image $C=G^{*}(C)$ equals the flat cone in $E_{\alpha}$ whose edges are the half-lines $D_{1}$ and $D_{2}$ respectively generated by $a_{1}=G^{\star}\left(A_{1}\right)$ and $a_{2}=G^{\star}\left(A_{2}\right)$. Due to $(5.7), C$ contains $-g ;$ put $b=a_{2}-a_{1}$. In view of the form (3.9), (3.10), (3.11) that relation $R \in \operatorname{cou}(U)$ takes in two-dimensional cases, the relation $s \in G^{*}(\operatorname{cou}(G(u)))$, holds between some elements $s \in E^{\star}$ and $u \in T$ if and only if

$$
\begin{aligned}
& s \in C \\
& s \in D_{1} \quad \text { if } \quad\langle b, u\rangle>0 \\
& s \in D_{2} \quad \text { if } \quad\langle b, u\rangle<0 .
\end{aligned}
$$

The dimension of $E_{\alpha}$ and $E_{\alpha}^{*}$ equals the freedom of $S_{\alpha}$. For picture clarity let us imagine it to be 3 ; through the choice of 
adapted bases in $\mathrm{E}_{\alpha}$ and $\mathrm{E}_{\alpha}^{\star}$, the positive definite matrix a may be made unit; this amounts to identify both spaces with a single copy of Euclidean $\mathbb{R}^{3}$ (at a more elaborate level it may be said that, due to the Riemannian structure of manifold $\gamma_{\alpha}$, the tangent and cotangent spaces at each point $q_{\alpha}$ merge into a single Euclidean linear space ; this standpoint is systematically used in [1], [7]).

Recall that we are now in the case where $\left\langle u_{i}^{\prime}, g\right\rangle$, i.e. the scalar product $u_{i}^{\prime} \cdot g$ in the sense of Euclidean $\mathbb{R}^{3}$, is strictly positive. Therefore the (non homogeneous) cone $u_{i}^{\prime}+C$ has nonempty intersection with the linear subspace $\mathrm{T}$;

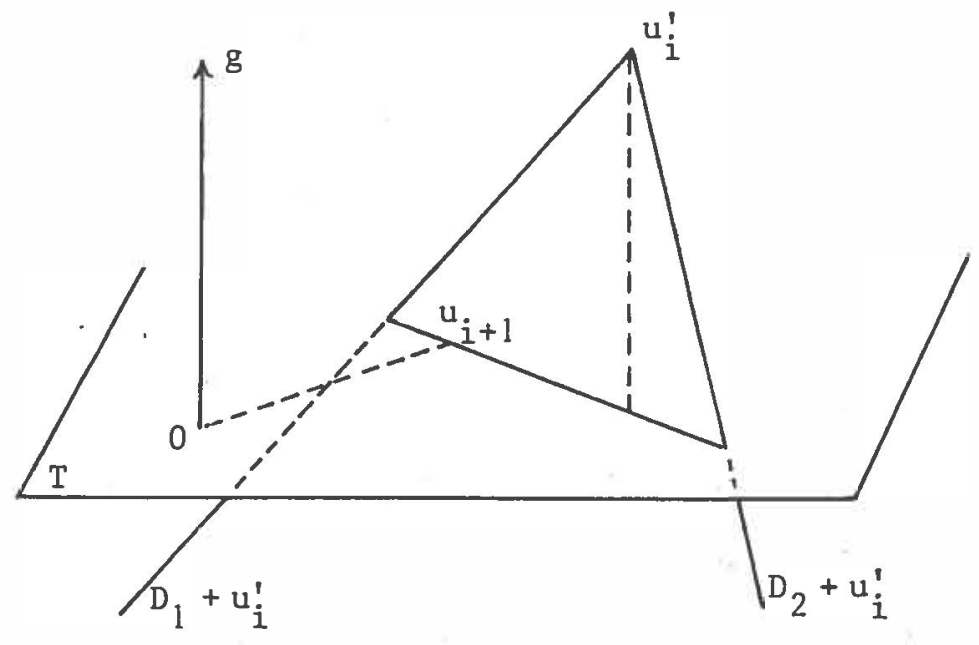

- Figure 1 -

this intersection is a line interval $\mathrm{S}$, possibly unbounded. Clearly in (5.9) and (5.10), if $u \in T$, the vector $b$ may equivalently be replaced by its orthogonal projection on $\mathrm{T}$; this is precisely a directing vector of $\mathrm{S}$. 
One readily concludes in the existence of a single point $u_{i+1} \in T$ satisfying (5.6), namely the nearest point in $S$ to the origin of $\mathbb{R}^{3}$; computing its coordinates is elementary.

For instance, in the case shown on Fig. $1, u_{i+1}$ happens to lie in the interior of $\mathrm{S}$; hence this vector is orthogonal to the plane of the flat cone C, i.e. $G^{\star}\left(E_{2}\right)$. This yields $G\left(u_{i+1}\right)=0$, meaning that the corresponding slip velocity is zero in that case.

REMARK 1 . Some elementary exercises in frictional Dynamics concern the motion of a mass-point on a surface. Exploiting Coulomb law in the traditional way requiresan expression of the normal component of acceleration : this classically involves velocity and the curvature tensor of the surface. Then one may be surprized at observing that our algorithms do not make use of the second derivatives of $f_{\alpha}$. Actually it turns out that curvature effects are completely accounted for by calculating the covector grad $f_{\alpha}$ at each step.

REMARK 2 . The above discretization procedure may be qualified as mixed implicit-explicit. A strong argument in favor of such a policy is that, in particular, it yields exactly zero velocity in cases where the initial conditions are those of equilibrium. This contrasts with some traditional approaches of dry friction ; for instance, in one dimensional situations, it is classical to express the law of dry friction through the function $\operatorname{sgn}(u)$, conventionally zero for $u=0$. Thereby, the multivalued character of Coulomb law is disregarded ; if explicit discretization schemes are derived from such formulations, they can only render equili- 
brium as a saw-tooth motion, with amplitude depending on the time-step. Also observe that, at each step of our algorithm, there is no difficulty in making the Coulomb mapping depend on $u_{i}$; this accounts in particular for the usual empirical distinction between the "static" and "dynamic" friction coefficients. 


\section{ALGORITHM BASED ON REACTIONS}

In presenting the main features of this algorithm we shall restrict ourselves to the case where the subsystems $S_{\alpha}$ are points, connected with springs and dashpots moving in a plane $0 x_{1} x_{2}$ (see fig.2). All of them are confined in the region $x_{2} \geqslant 0$. The obstacle $B_{0}$ has rectilinear boundary $x_{2}=0$, moving along the $x_{1}$ axis at velocity $E$. We consider small displacements so that the equation (2.5) governing the system may be written as

$$
m^{\alpha} \ddot{q}_{\alpha}+v^{\alpha}(\dot{q})+k^{\alpha}(q)=p_{\alpha}(t)+R_{\alpha}, q_{\alpha}=\left(q_{\alpha}^{1}, q_{\alpha}^{2}\right) \text {. }
$$

$\mathrm{V}_{\alpha}(\dot{\mathrm{q}})$ represents the viscosity forces from the dashpots; $\mathrm{K}_{\alpha}(\mathrm{q}) \mathrm{re}^{-}$ presents the elastic forces from the springs; $P_{\alpha}(t)$ is some force explicitely given as a function of time. $R_{\alpha}$ is written only if $\alpha$ is the index of a point in contact with the obstacle. In that case $G_{\alpha}^{\star}$ is nothing but the identity mapping from $E_{2}$ onto itself. The above equations may also be written as

$$
M \ddot{q}+V \dot{q}+K q=P(t)+G{ }^{*} R \quad,
$$

where the inertia matrix $M$ is diagonal; $V$ and $K$ are symmetric ronm:islive matrices. Let us denote by $n$ the number of points and $x$ the 
number of contact points, assuming that for some interval of time, these points remain in contact. Contact points are indexed by $\beta \in\{1, \ldots, x\}$ and they have a corresponding index in the $\alpha$ list, that we denote $\alpha_{\beta}$. The $2 x$ vector $R$ is the column of the $x$ two-dimensional vectors $R_{\alpha}$. $\mathrm{G}^{\star}$ is thus a mapping from $\mathbb{R}^{2 x}$ into $\mathbb{R}^{2 \mathrm{n}}$, with the identity matrix on the $\alpha_{\beta}, \beta$ block and zero elsewhere.

There is a wide range of methods to compute an approximation of the solution to an ordinary differential equation such as (6.1). In numerous cases, explicit or implicit methods provide two matrices $\mathbb{A}_{h}, \mathbb{B}_{h}$, (h denotes the step length $t_{i+1}-t_{i}$ ) deriving an approximate value $\mathrm{q}_{i+1}$ of $\mathrm{q}\left(\mathrm{t}_{i}\right)$ and $\dot{\mathrm{q}}_{\mathrm{i}+1}$ of $\dot{\mathrm{q}}\left(\mathrm{t}_{i+1}\right)$ from an approximate value $\mathrm{q}_{i}$ of $q\left(t_{i}\right)$ and $\dot{q}_{i}$ of $\dot{q}\left(t_{i}\right)$ namely

$$
\begin{aligned}
& {\left[\begin{array}{c}
q_{i+1} \\
\dot{q}_{i+1}
\end{array}\right]=A_{h}\left[\begin{array}{c}
q_{i} \\
\dot{q}_{i}
\end{array}\right]+\mathbb{B}_{h} h \bar{\varphi}} \\
& \bar{\varphi}=\bar{P}+G^{\star} \bar{R}
\end{aligned}
$$

$\bar{P}$ is an approximant of $P$ on $\left[t_{i}, t_{i+1}\right]$ and $\bar{R}$ is an approximant of $R$ on $\left[t_{i}, t_{i+1}\right]$. One has also to choose the values $\bar{q}$ and $\bar{q}$ of approximants of $q$ and $\dot{q}$ on $\left[t_{i}, t_{i+1}\right]$, in order to get an approximant of the slip velocity on $\left[t_{i}, t_{i+l}\right]$. A possible choice is

$$
\begin{aligned}
& \bar{q}=(1-s) q_{i}+s q_{i+1} \\
& \bar{q}=(1-s) \dot{q}_{i}+s \dot{q}_{i+1} \quad s \in[0,1]
\end{aligned}
$$


The choice $s=1$ proves the best one regarding stability or smoothing effects. The velocity $\dot{q}_{i+1}$ resulting from (6.2) may be written as

$$
\dot{q}_{i+1}=\Gamma+B^{\prime} h G^{*} \vec{R} \quad \text {. }
$$

Here $\Gamma$ contains terms with $q_{i}, \dot{q}_{i}$ and $\bar{P}$. The term $G^{\star} \bar{R}$ can be decomposed into

$$
G^{\star} \bar{R}=G_{T}^{*} \bar{R}_{T}+G_{N}^{*} \bar{R}_{N}
$$

where $\bar{R}_{T}$. is the $x$ column of tangential components of $\bar{R}$ and $\bar{R}_{N}$ the $x$ column of normal components of $\bar{R}$. Actually $G_{T}$ and $G_{N}$, the transpose matrices of $G_{T}^{\star}$ and $G_{N}^{*}$, are projectors from $\mathbb{R}^{2 x}$ on $T_{\alpha_{1}} \times T_{\alpha_{2}} \times \ldots \times T_{\alpha x}$ and from $\mathbb{R}^{2 x}$ on $N_{\alpha_{1}} \times N_{\alpha_{2}} \times \ldots \times N_{\alpha x}$, respectively. Let us express that the velocity $\left(\dot{q}_{\alpha}\right)_{i+1}$ lies in the tangent space $T_{\alpha}$ :

$$
0=G_{N} \dot{q}_{i+1}=G_{N} \Gamma+G_{N} B^{\prime} G_{T}^{\star} h \bar{R}+G_{N} B^{\prime} G_{N}^{\star} h \bar{R}_{N}
$$

Since in most cases $B^{\prime}$ is an invertible matrix (in fact $B$ is close to M), $G_{N} B^{\prime} G_{N}^{*}$ is invertible and the above equation yields an expression of $R_{N}$ as a function of $R_{T}$. The slip velocity expresses as

$$
G_{T} \dot{q}_{i+1}-[E]=U=G_{T} \Gamma+G_{T} B^{\prime} G_{T}^{*} h_{T} \bar{R}_{T}+G_{T} B^{\prime} G_{N}^{*} h \bar{R}_{N}
$$

By $[E]$ is denoted the $X$ column with components $E$, the velocity of the moving wall. The matrix $G_{T} E^{\prime} G_{T}^{\star}$ is symmetric positive definite. Inequalities 
are tested. The inclusion

$$
-u_{\alpha} \in \partial \psi\left(k_{N_{\alpha}}\right)
$$

may be equivalently transformed into the following fixed point condition

$$
R_{T_{\alpha}}=\operatorname{proj}_{D_{\alpha}}\left(R_{T_{\alpha}}-o\left(W_{\alpha}+G_{T^{B}} G_{T}^{\star} \text { h } \bar{R}_{T}\right)\right)
$$

with $D_{\alpha}=R_{N_{\alpha}} D_{1}$ and

$$
W=G_{T} \Gamma+G_{T} B^{\prime} G_{N}^{\star} h \vec{R}_{N} \text {, }
$$

Observe that the impulse $h \bar{R}$ emerges as the natural variable. We shall develop in section 7 below how this allows for treating shocks as well as smooth motions. Of course the method applies to more general situations than those related to systems of points. The projectors have to be computed at each step. Nevertheless in the case of a plane obstacle the computation may be avoided in checking whether the set of contact points at some step is the same set as the one met at the previous step. If a motion with persistent contact is expected then the projectors may be computed once for all. The algorithms may be constructed in taking advantage of simplifications allowed by special forms of the matrix $M$ and of the projectors. For instance $M$ or $G_{N} B^{\prime} G_{T}^{*}, G_{N} B^{\prime} G_{N}^{*}, G_{T} B^{\prime} G_{T}^{*}$ may be diagonal matrices (see for instance [12]).

The system of figure 3 is composed of ten material points. The points are connected together by viscoelastic dipoles (involving spring and dashpot). Each of them is submitted to a constant vertical force (gravity) and is pushed against the obstacle by a viscoelastic dipole. The angle of rake is such that when a point moves to the right, the pressure against 
the obstacle increases. For this system one may observe a motion with persistent contact. The velocities and tangential components of the reaction as functions of time are plotted for points $1,3,5$ on figure 3. A stick-slip phenomenon is in evidence for points 3 and 5 (see arrows $\mathbf{V})$. The velocity settles at the value $E$ of the obstacle velocity while a drop of the tangential component of the reaction occurs. Figure 4 shows a similar system except for the angle of rake of the dipoles pushing against the obstacle. When a point moves to the right, the pressure against the obstacle decreases. The $x_{1}$ and $x_{2}$ coordinates of the points $1,2,3,4,5$ as functions of time are plotted on figure 4 . One sees (arrow - ) that the point 3 leaves smoothly the obstacle. Then the point comes back into contact (arrow - ) with a shock after which it slides again. By plotting velocity one could see that stick-slip is also present in this case.
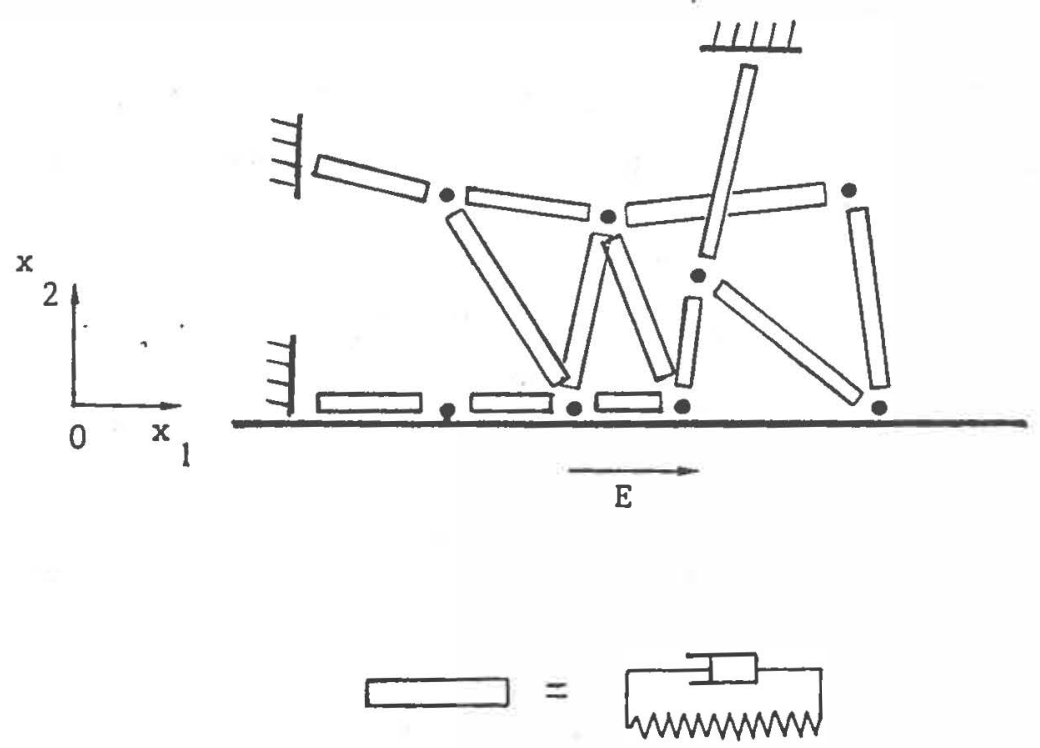

- Figure 2 - 

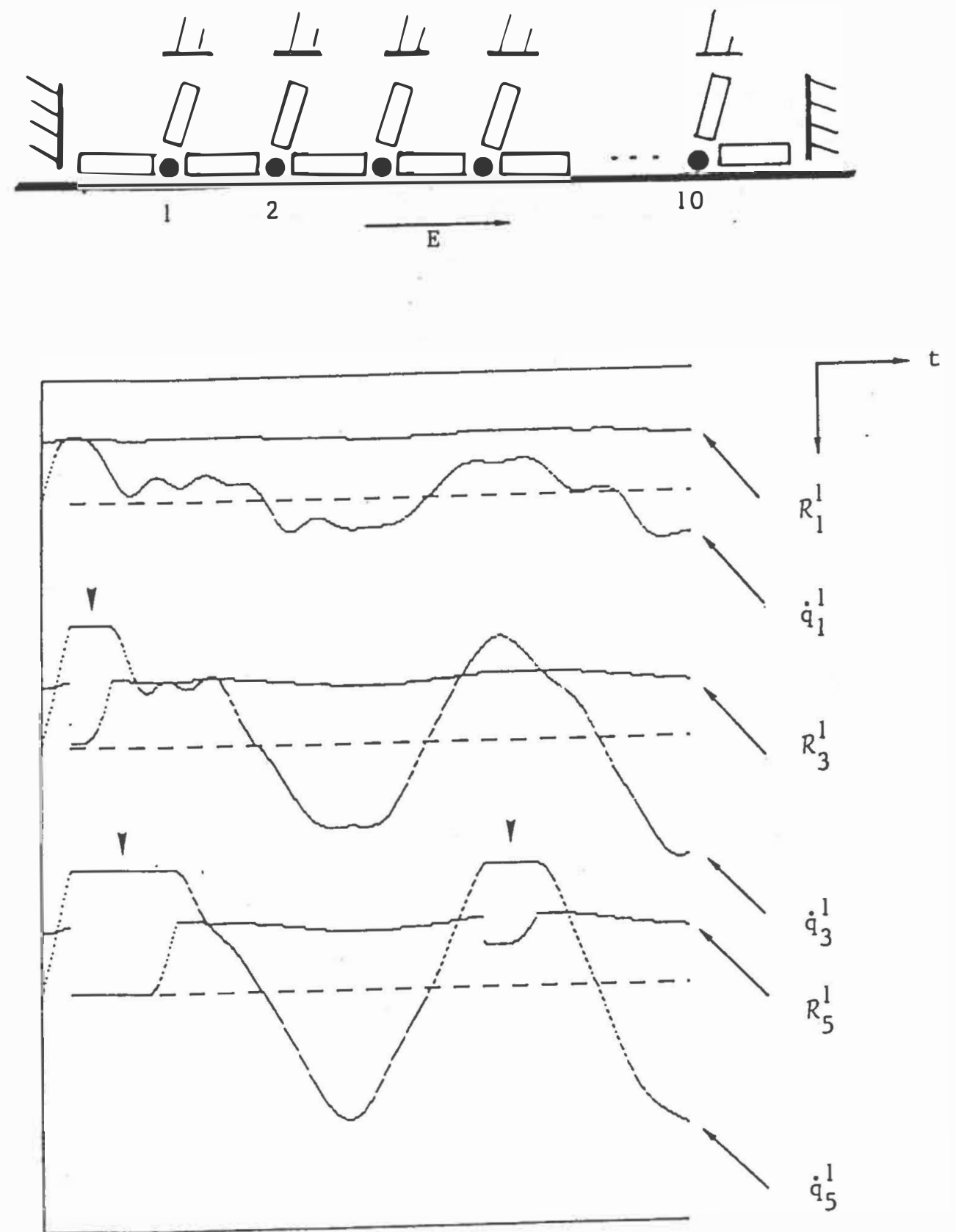

- Figure 3 - 

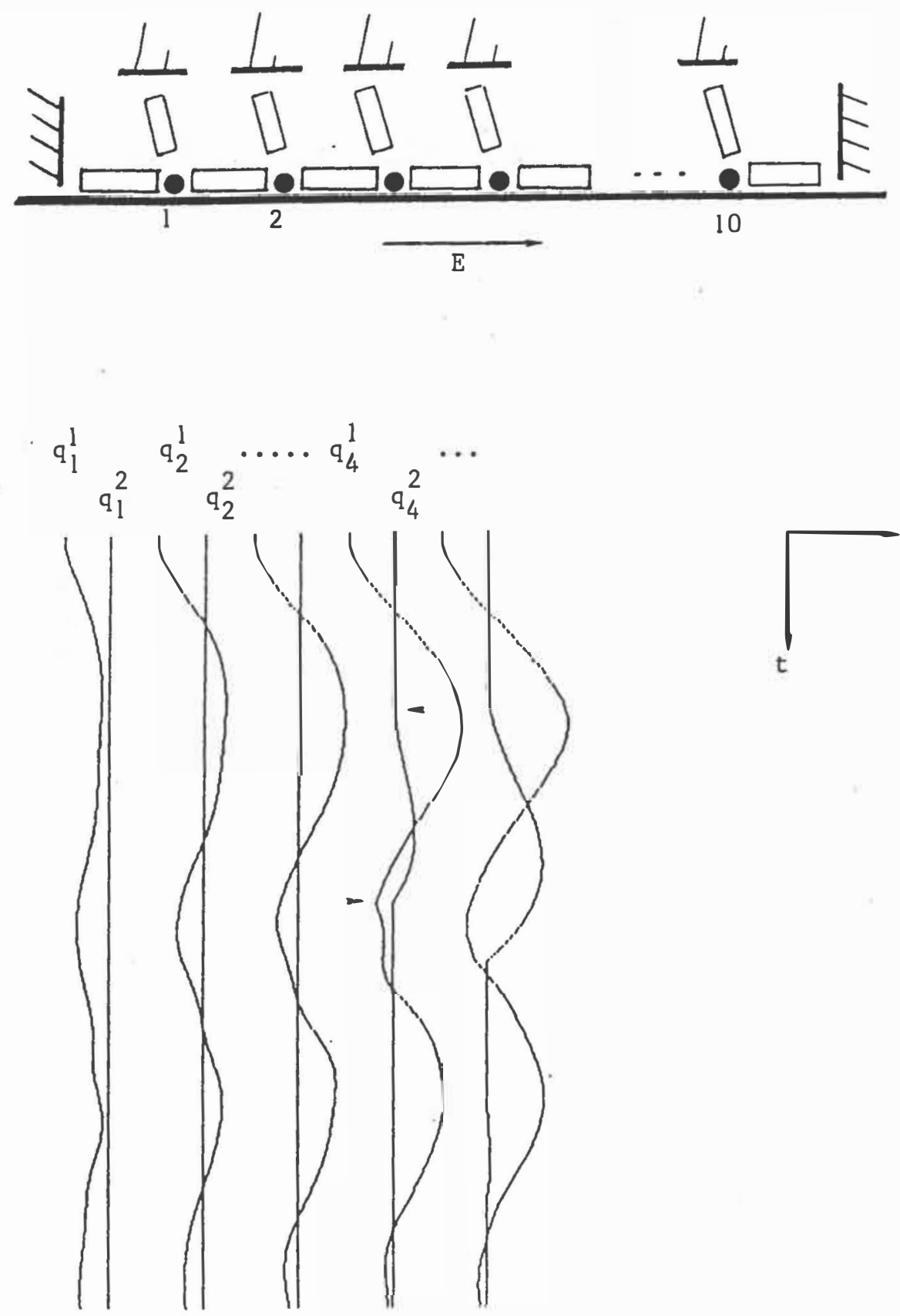

- Figure 4 - 


\section{SHOCK CALCULATION}

All what precedes concerns motions smooth enough for the second derivative $\ddot{q}$ to exist. Such motions may verify $f_{\alpha}\left(t, q_{\alpha}\right) \leq 0$ either as a strict inequality or as an equality. In particular, some episode with persistent equality may be followed by contact break without any jump in velocity.

On the contrary, if an episode with $\mathrm{f}_{\alpha}<0$ ends at some instant $t_{s}$ such that $f\left(t_{s}, q_{\alpha}\left(t_{s}\right)\right)=0$, the continuity of $\dot{q}_{\alpha}$ at $t_{s}$ cannot be expected in general. We shall assume the existence of the right-limit $u_{\alpha}^{+}=\dot{q}_{\alpha}\left(t_{s}^{+}\right)$and of the left-limit $u_{\alpha}^{-}=\dot{q}_{\alpha}\left(t_{s}^{-}\right)$.

This is a shock, the physical circumstances of which are inevitably intricate. Some insight can only be gained from relaxing, at least qualitatively, the idealization previously adopted. A certain amount of deformation will be accepted for the bodies in presence, so that the sudden velocity change develops through a little longer duration than the single instant $t_{s}$. By equalling the integrals of both members of the equations of Dynamics over this duration, one classically obtains an expression for the momentum change in terms of the percussion of constraint.

In the no-friction case, it is usually admitted that this percussion is normal to the colliding surfaces (this might be discussed; see e.g. 
[11], sect. 9.7.c, Remarque). Even so the above balance of momentum does not provide enough information to determine $u_{\alpha}^{+}$. The complementary condition is commonly derived from assuming that the shock is elastic or, alternatively, inelastic.

Let us restrict ourselves here to the same simple case as in previous sections : the system is supposed scleronomic and the function $f_{\alpha}$ does not depend on time. Then the definition of an elastic shock simply reduces to asserting that the kinetic energy of the system is preserved, while inelastic shock is characterized by condition $\left\langle\mathrm{u}_{\alpha}^{+}\right.$, grad $\left.\mathrm{f}_{\alpha}\left(\mathrm{q}_{\alpha}\right)\right\rangle=0$. One proves that the latter essentially involves energy dissipation (cf. [1], where the concept of an inelastic shock is also extended to the case of constraints expressed by several inequalities).

In practice, physical arguments concerning the energy balance of a shock are generally uncertain. Even if the slighlty deformable material of which the system is built may safely be treated as elastic, one cannot a priori assert energy preservation. In fact the slight deformation resulting from the impact is not usually restricted to the small zone of contact ; it propagates to the whole of colliding parts and possibly also to other connected bodies, whether internal or external to the system. Therefore, after bounce, various parts may remain in a state of vibration which, at the macroscopic scale, amounts to energy loss.

In contrast, it seems easier to characterize some physical situations where the inelasticity of shocks is a pretty safe guess. A familiar example is that of a wood or metal ball landing on a floor of beaten earth. Frictional effects between the colliding bodies still complicate the 
situation. A theory of frictional shock was proposed by G. Darboux and further improved by J. Pérès (see e.g. [3], p. 316-328). It consists in studying the evolution of the velocity as a function of some "microscopic time" ordering the very short duration of the shock, while position is treated as constant. This is interesting as an early example of a multiple scaling method but the physical relevance seems difficult to assess ; in particular the estimation of shock end proves critical.

We shall adopt a cruder approach of frictional shock by assuming that the percussion of contact relates to the final velocity $u_{\alpha}^{+}$, through the same generalized Coulomb law as does the force of contact in smooth motions.

The mathematical background of "nonsmooth Dynamics" has been formulated in [1] under the assumption that velocity $u$ is a function of $t$ with locally bounded variation, non differentiable in general. Then the role of the acceleration is played by the differential measure du, a vector measure on the considered time interval. Accordingly, forces are replaced by vector measures, comprising possible percussions ; in smooth cases these measures admit, relative to the Lebesgue measure $\mathrm{dt}$, some density functions whose values are forces in the proper sense.

In that context, the differential inclusion (5.2) receives a precise generalized meaning. It turns out that the discretization algorithms of sections 5 and 6 yield approximate solutions of the corresponding problems through exactly the same computation procedures as in smooth cases. If some discretization interval $\left(t_{i}, t_{i+1}\right)$ happens to include shock, it only comes that the difference $u_{i+1}-u_{i}$ has no more the same 
order of magnitude as $h=t_{i+1}-t_{i}$; this difference approximates the integral of the measure du over the considered time interval.

Thus the decision of relating frictional percussion to the rightlimit of velocity appears to parallel exactly the choice previously made of an implicit discretization scheme in treating the differential inclusion, as well as the assumption of shock inelasticity.

In our opinion, this approach has the following methodological virtues. It standardizes a type of irreversible process, resulting in evolution problems with strong theoretical and numerical consistency. Some practical instances certainly exist where the assumptions made are in satisfactory agreement with reality. But, above all, this ideal situation provides a safe basis to which further empirical corrections could be added in rational order. This looks similar, for instance, to the concept of standard dissipative process in thermoplasticity [13] or to the use of Lax's entropy conditions when studying shockwaves in hyperbolic conservation laws (see e.g. [14]). 
8. AN EXAMPLE OF OSCILLATOR

WITH FRICTIONAL UNILATERAL CONTACT

Figures $6,7,8$, have been produced by a computer program using the algorithm of sect. 5 .

A rigid body $B$ performs a motion parallel to the $(x, y)-p l a n e$. The plotter draws the profile of $B$ in its initial position : the significant part of it consists of an arc of circle with center $A$ and radius $b$.

Using as position parameters the coordinates $\mathrm{X}, \mathrm{Y}$ of the center of mass $G$ and some rotation angle $\Theta$, one gives to the kinetic energy of $B$ the form (2.3). In this computation $b=m=I=1 ; A G=1,732$. The body is confined in the region $y \geqslant 0$; its possible contact with the fixed wall $y=0$ involves dry friction with coefficient 0.5 .

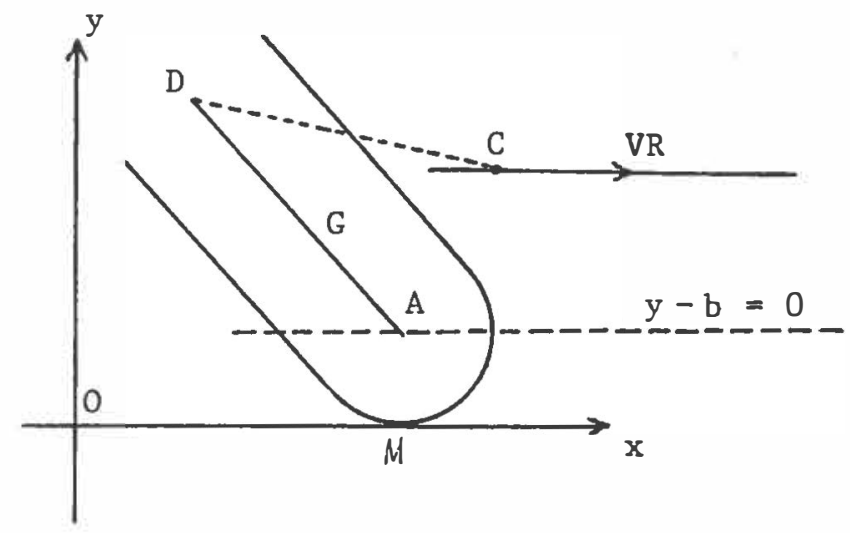

- Figure 5 - 
Here are the driving forces :

$1^{\circ}$ Some particle $D$ of $B$ (located in this computation on the line AG, with $G D=1.6$ ) experiences a linearly elastic pull-back, centered at some external point $C$ with prescribed uniform motion : $C$ is supposed to move along the line $y=2.7$ with velocity 30 to the right. The pull-back force has its $(x, y)$-components proportional to the corresponding components of vector DC ; the respective stiffness coefficients are 200 for the $x$-component and 2000 for the $y$-component. $2^{\circ}$ In addition, a constant torque $C_{r}$ is exerted on $B$. In the figured case, negative values of $C_{r}$ tend to favor the contact of $B$ with the wall.

Steady oscillations of the system, corresponding to three values of $\mathrm{C}_{\mathrm{r}}$ are presented. The plotter draws, at constant time intervals, a line with origin $A$ and directed to $G$. The unilateral constraint makes $A$ lie in half-plane $y-b \geqslant 0$; this point belongs to the boundary line $y-b=0$ whenever $B$ keeps contact with the wall. Numbers refer to steps of the time-discretization algorithm.

Figure 6 is obtained for $C_{r}=-700$; discretization step length 0.002 ; position plotted every 4 th step. Then $B$ does not get loose from the wall. One observes angular oscillations ; also oscillations with longer period affect the space-lag from the pull-back center, as measured for instance by the variable $l=x(C)-x(G)$. The program might be used to draw additionally the two-dimensional trajectories concerning any couple among the 6 variables $\ell, Y, \theta, \dot{\imath}, \dot{Y}, \dot{\theta}$; this would display the typical pattern of a "doubly periodic" motion, revealing 
that the trajectories in $\mathbb{R}^{6}$ are coiled on some invariant torus. In fact, the present circumstances : persistent contact and slip velocity with constant direction make the motion verify as well the equations of smooth, bilateral dynamics. Therefore the system behaves like a classical smooth nonlinear oscillator.

Figure 7 corresponds to $C_{r}=-200$; step length 0.001 ; position plotted every 8 th step. The motion, in variables $\ell, Y, \Theta$ is now periodic. Each period consists of a contact-free episode, ending with a shock of $B$ against the wall. The program is also able to display the slip velocity : its value following the shock is found zero; the body gets loose immediately from the wall in a smooth way.

Figure 8 corresponds to $C_{r}=-1.00$; step-length 0.0005 ; position plotted every 15 th step. The motior is again periodic but with some more complicated pattern. Every period exhibits two different episodes of contact-free motion. An episode of the first sort ends with a shock, followed by a nonzero time-interval with persistent contact and positive slip velocity. Afterwards the body $B$ gets loose from the wall with zero normal velocity and performs the second sort of contact-free episode. This ends with a second shock, immediately followed by an episode of the first sort. The slip velocity resulting from the second shock is positive, but the normal velocity is zero, in agreement with our inelasticity assumption. 

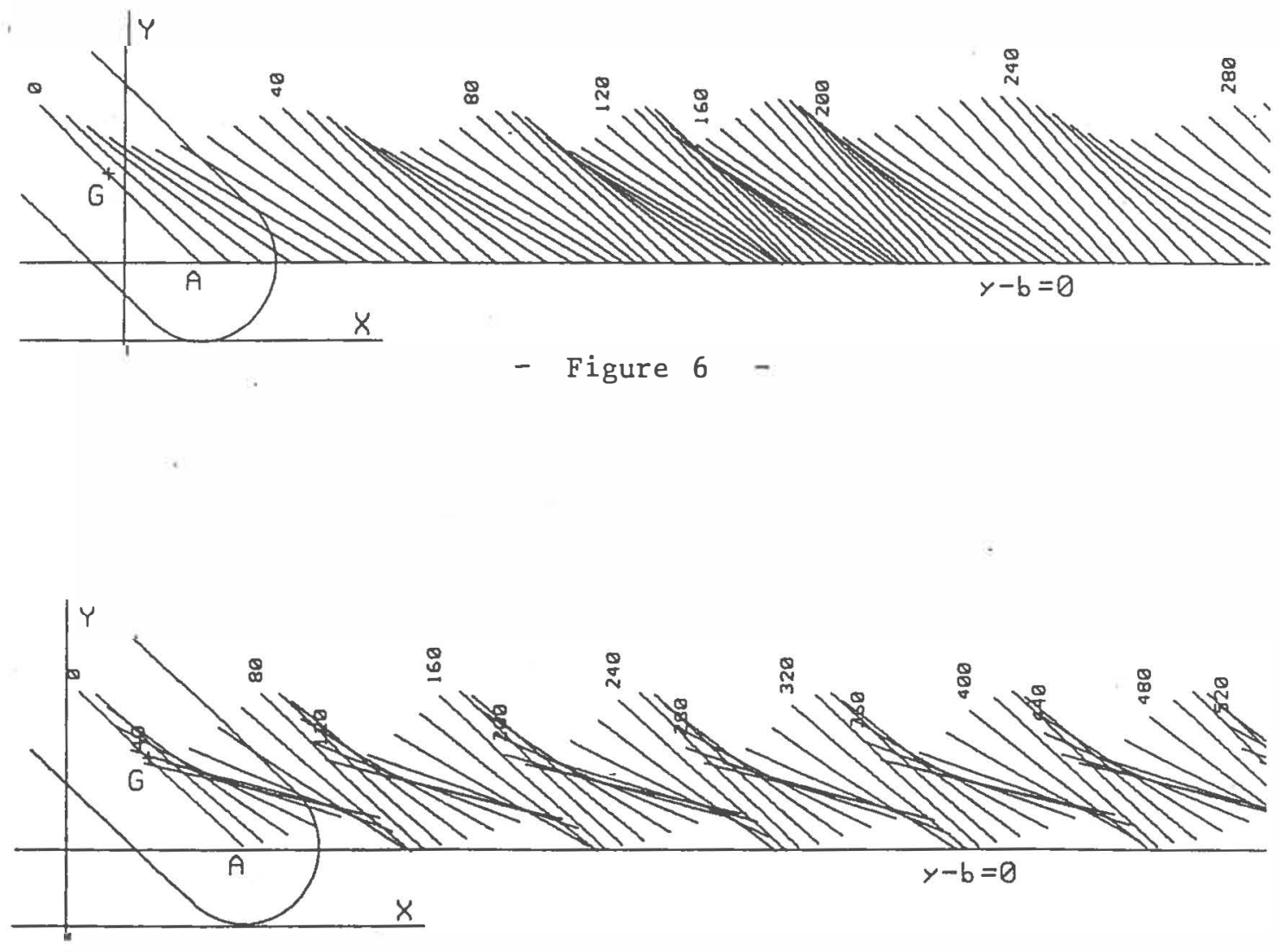

- Figure 7 -

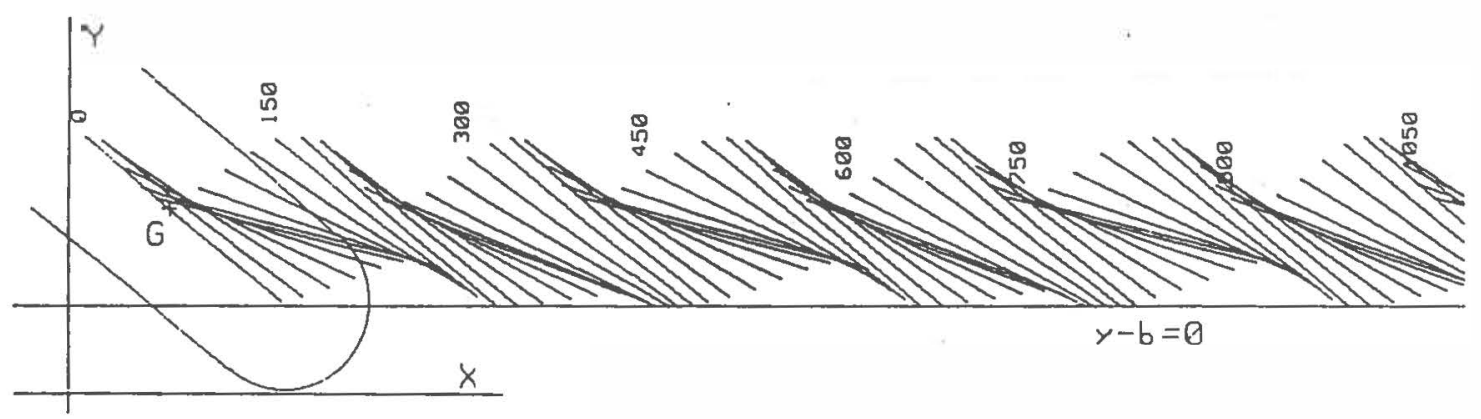

- Figure 8 - 
REFERENCES

1. Moreau, J.J., Standard inelastic shocks and the dynamics of unilateral constraints, in : Unilateral Problems in Structural Analysis, CISM Courses and Lectures $\mathrm{n}^{\circ} 288$ (Ed. G. De1 Piero and F. Maceri), Springer-Verlag, Wien-New York 1985, 173-221.

2. Delassus, E., Considérations sur le frottement de glissement, Nouv. Ann. de Mathématiques, 4ème Ser., 20 (1920), 485-496.

3. Pérès, J., Mécanique Générale, Massoı, Paris, 1953.

4. Jean, M. and Pratt, E., A system of rigid bodies with dry friction, Int. J. Engng. Sci., 23 (1985), 497-513.

5. Moreau, J.J., Sur les lois de frottement, de plasticité et de viscosité, C.R. Acad. Sci. Paris, Sér. A, 271 (1970), 608-611.

6. Moreau, J.J., Application of convex analysis to some problems of dry friction, in : Trends in Applications of Pure Mathematics to Mechanics, vo1. II (Ed. H. Zorski), Pitman, London-San Francisco-Melbourne 1979, 263-280.

7. Moreau, J.J., Dynamique de systèmes à liaisons unilatérales avec frottement sec éventuel; essais numériques, Université des Sciences et Techniques du Languedoc, Montpellier, Lab. de Mécanique Générale des Milieux Continus, Note Technique 85-1, 1985. 
8. Lötstedt, P., Coulomb friction in two-dimensional rigid body systems, Z. Angew. Math. u. Mech. 61 (1981), 605-615.

9. Lötstedt, P., Mechanical systems of rigid bodies subject to unilateral constraints, SIAM J. Appl. Math. 42(1982), 281-296.

10. Moreau, J.J., On unilateral constraints, friction and plasticity, in : New Variational Techniques in Mathematical Physics, CIME $2^{\circ}$ ciclo 1973, (Ed. G. Capriz and G. Stampacchia), Edizioni Cremonese, Roma, $1974,175-322$.

11. Moreau, J.J., Mécanique classique, vol. 2 Masson, Paris, 1971.

12. Jean, M., Un algorithme numérique simple pour un système d'oscillateurs avec frottement de Coulomb sur un plan, Université des Sciences et Techniques du Languedoc, Lab. de Mécanique Générale des Milieux Continus, Note Technique 85-2, 1985.

13. Halphen, B. and Nguyen, Q.S., Sur les matériaux standards généralisés, J. de Mécanique, 14, 39, 1975.

14. Lax, P.D., Hyperbolic systems of conservation laws and the mathematical theory of shock waves, Regional Conf. Sér. in Appl. Math., $n^{\circ} 11$, S.I.A.M., Philadelphia, 1973. 